\title{
Project 90256
}

\section{Multipurpose Radiation Resistant Semiconductor Detectors for Alpha, Neutron \& Low Energy Gamma Ray Measurements at High Temperatures in High-Intensity Gamma Ray}

\author{
Ruddy, Frank H. \\ Westinghouse Electric Company LLC
}

RESULTS TO DATE: PROGRESS REPORT DOE Grant DE-FG02-04ER63734 Frank H. Ruddy, Principal Investigator

\subsection{Summary}

Work scheduled under year two of DOE Grant DE-FG02-04ER63734 is on schedule and all year-two milestones have or will be met. Results to date demonstrate that unprecedented silicon carbide (SiC) energy resolution has been obtained, and that SiC detectors may achieve energy resolution that exceeds that obtainable with the best silicon alpha spectrometers. Fast-neutron energy spectrometry measurements indicate that recoil-ion energy spectrometry should be possible with $\mathrm{SiC}$ detectors. Furthermore, SiC detectors have been demonstrated to perform well even after gamma-ray exposures of 1.E09 Rad. This result and the previously demonstrated capability of SiC detectors to operate in elevated-temperature environments are very promising for potential DOE EMSP applications. A new class of multipurpose, radiation-resistant semiconductor detectors that can be used in elevated-temperature and high-radiation environments is being developed under this grant. These detectors, based on silicon carbide ( $\mathrm{SiC}$ ) semiconductor are designed to have larger active volumes than previously available $\mathrm{SiC}$ detectors, and are being tested for their response to alpha particles, X-rays and low energy gamma rays, and fast neutrons. Specifically, $\mathrm{SiC}$ radiation detectors with larger areas and 100-micrometer thick active regions have been designed and manufactured according to detector-design specifications. Detectors based on a Schottky diode design were specified in order to minimize the effects of the detector entrance window on alpha particle measurements. During manufacture of the Schottky diodes, the manufacturer also provided a set of large-volume $\mathrm{SiC} \mathrm{p-i-n} \mathrm{diodes} \mathrm{for} \mathrm{testing} \mathrm{Extensive} \mathrm{alpha} \mathrm{particle} \mathrm{measurements} \mathrm{have} \mathrm{been} \mathrm{carried} \mathrm{out} \mathrm{to}$ test and quantify the response of the SiC Schottky diodes. Exposures to 148-Gd, 213-Po, 217-At, 221-Fr, 225-Ac, 237-Np, 238-Pu, 240-Pu, and 242-Pu sources were used to obtain detailed alpha response data in the alpha energy range from $3182.787 \mathrm{keV}$ to $8375.9 \mathrm{keV}$. The 148-Gd, 213-Po, 217-At, and 221-Fr sources provide energy-separated, mono-energetic alpha particle peaks which can be analyzed to provide detailed information on the energy response characteristics of the detectors. As was reported last year, a highly linear response was obtained between observed pulse height and alpha-particle energy over the entire energy range. Detailed full width at half maximum (FWHM) measurements were made for each of five mono-energetic peaks. The FWHM values ranged from $41.5 \mathrm{keV}$ for 3182.787-keV 148-Gd (1.3\% energy resolution) to $55.4 \mathrm{keV}$ for $8379.5-\mathrm{keV}$ 213-Po (0.66\% energy resolution). Although these energy resolution values are comparable to those obtainable with silicon alpha-particle spectrometers and surpass the best values reported previously for $\mathrm{SiC}$ detectors, other factors in addition to the inherent SiC energy resolution contribute to the observed values and were evaluated. Details of the energy deposition processes that contribute to the FWHM were modeled with calculations using the SRIM-2003.26 code developed by Ziegler and Beirsack. Electronic and statistical broadening of the FWHM were also evaluated in order to isolate the component of the FWHM that is inherent to SiC semiconductor. It was found that the SRIM calculations systematically overestimated the range straggling component of the FWHM leading to calculated values that exceeded the measured total FWHM values. It is believed that the overestimates are a result of inherent limitations of the SRIM code. The range-straggling component of the measured energy resolution results primarily from energy-loss processes in the detector entrance window, which consists of thin layers of $\mathrm{Au}, \mathrm{Pt}$, and Ti. Therefore, it was decided to perform measurements to evaluate the range straggling component directly. For this purpose, a high-resolution (15 keV FWHM) Si alpha spectrometer 
was obtained. The energy resolution values were carefully evaluated for this detector using the same sources that were used for the SiC detectors, and a follow-on measurement of these FWHM values will be made after $\mathrm{Au} / \mathrm{Pt} / \mathrm{Ti}$ layers identical to those on the $\mathrm{SiC}$ detector have been applied to the Si detector. The measured FWHM differences in the Si detector will be used to evaluate the range-straggling contribution for the $\mathrm{SiC}$ detectors. It is anticipated that the FWHM component inherent to SiC may be less than $20 \mathrm{keV}$, which is comparable to the best available silicon alpha particle detectors. Irradiations are continuing in the Westinghouse Gamma Hot Cell to assess the performance of SiC radiation detectors after intense gamma irradiation. To date, only negligible changes in detector response have been observed following cumulative 137Cs gamma-ray doses of 1.4E09 Rad (14 MGy). Conventional silicon detectors suffer severe response deterioration at about E06 Rad. Therefore, SiC detectors are far more resistant to gamma radiation effects than silicon detectors. These measurements will be continued over the three-year duration of the program resulting in cumulative gamma ray exposures up to 4E09 Rad. Initial neutron exposures using p-i-n diodes with 4-mm x 4-mm x 90-micrometer active volumes leading to excellent fast neutron response results were reported last year. These measurements have been extended to SiC Schottky diodes with 4.4-mm x 4.4-mm x 100-micrometer and 6.0-mm x 6.0-mm x 100-micrometer active volumes. Exposures to $14-\mathrm{MeV}$ neutrons from an electronic D-T source show prominent and well-resolved reaction peaks from 12-C(n,alpha)9-Be and 28-Si(n,alpha)25-Mg reactions in the $\mathrm{SiC}$ detector. Ten peaks are observed for the latter reaction corresponding to branches to the ground state and excited states of 25Mg. The energies of these peaks can be used to accurately determine the energy of fusion neutrons from a D-T plasma, and the widths of the peaks can be used to determine the ion plasma temperature. Additional exposures of these p-i-n detectors to 252-Cf , 235-U(n,f) and 241-Am-Be isotopic neutron sources have also been carried out. In addition, D$\mathrm{T}$ angular distribution measurements have been carried out to quantify the effects of the kinematic neutron energy shift with laboratory observation angle on the observed neutron reaction peaks in SiC. Modeling of the neutron response of $\mathrm{SiC}$ detectors is proceeding in order to provide spectrum de-convolution methods to infer the incident neutron energy spectrum from the reaction product recoil ion pulse height spectrum. The goal of the modeling is to provide a means to perform fast neutron energy spectrometry measurements with $\mathrm{SiC}$ detectors.

\subsection{Status of Schedule \& Milestones}

The status of the tasks and milestones that were scheduled for years one and two are summarized in the following sections.

Task 1. Design of SiC Radiation Detectors Task 1 was completed ahead of schedule in December 2003.

Task 2. Fabrication of the Multipurpose SiC Detectors Task 2 was completed on schedule in June 2004.

Task 3. SiC Detector Testing Subtask 3.1 Gamma-ray radiation effects testing. SiC Schottky diodes are being subjected to long-term gamma-ray exposures in the Westinghouse Gamma Hot Cell. To date, only negligible changes in the $\mathrm{SiC}$ detector response to alpha particles have been observed following gamma-ray doses up to 1.4E09 Rad. These diodes will be subjected to continuing periodic response testing at gammaray doses up to 4E09 Rad in order to assess any effects of gamma ray exposure on detector performance.

Subtask 3.2 Charged-Particle Response Testing 3.2.1 Alpha Response Testing Testing of the SiC Schottky diodes with 148-Gd, 213-Po, 217-At, 221-Fr, 225-Ac, 237-Np, 238-Pu, 240-Pu, 239-Pu, and 242-Pu alpha particles has been completed. Excellent energy resolution results have been obtained in the testing. Measured peak shapes for the mono-energetic peaks from 148-Gd, 213-Po, 217-At, and 221-Fr are being analyzed to determine the components of the peak full width at half maximum (FWHM) that are due to energy straggling in the detector Schottky contact (entrance window), electronic noise, charge carrier statistics, and processes inherent to SiC semiconductor. The milestone, ?Complete SiC Detector AlphaResponse Testing?, scheduled in for December 2004 was completed ahead of schedule in October 2004. 3.2.2 Alpha Response Modeling Detailed range-energy calculations were carried out to assess the effects of the $\mathrm{SiC}$ contact layers and counting geometry on the energy deposition processes in the $\mathrm{SiC}$ detectors. Range straggling is a major contributor to the broadening of the observed alpha particle peaks in SiC, because gold, platinum, and titanium metallic layers are required to ensure good bonding of electrical leads 
to the SiC Schottky contact, which also serves as the detector entrance window. However, it was found that the range-energy code SRIM systematically overestimates the straggling component. Therefore, the straggling component is being measured directly by measuring the energy resolution values for a highresolution Si alpha detector before and after the application of $\mathrm{Au} / \mathrm{Pt} / \mathrm{Ti}$ layers identical to those applied to the SiC detectors. These Si measurements coupled with already completed measurements of the noise broadening of the $\mathrm{SiC}$ peaks will enable the peak resolution component inherent to the properties of $\mathrm{SiC}$ to be isolated and evaluated.

Subtask 3.3 X-Ray and Low-Energy Gamma-Ray Response Testing X-ray response tests are being carried out using 137-Cs(137m-Ba), 207-Bi, 241-Am and 237-Np sources. Completion of these tests is anticipated before the end of year 2 in order to meet the milestone ?Complete Low-Energy Gamma and X-ray response Testing?.

Subtask 3.4 X-Ray and Low-Energy Gamma-Ray Response Modeling This task is in progress.

Subtask 3.5 High-Energy Neutron Response Testing The SiC p-i-n diode tests that were reported last year were supplemented with SiC Schottky diode neutron response measurements. The 12-C(n,alpha)9-Be peak and multiple peaks corresponding to the 28-Si(n,alpha)25-Mg ground state and excited state branches were observed both with the p-i-n and Schottky diodes. Peak broadening resulting from pulse-height defect effects was modeled using SRIM calculations. Angular distribution measurements were taken with $\mathrm{SiC}$ Schottky diodes in order to use the kinematic energy shift to determine the energy scale for the 12C(n,alpha)9-Be reactions. 252-Cf and 241-Am-Be neutron response spectra were taken with both the SiC p-i-n and Schottky diodes. Additional 235-U(n,f) neutron response measurements have been carried out for comparisons with the 252-Cf fission neutron measurements. D-D response testing for 2.5-MeV neutrons will be completed before the end of year 2 to complete all scheduled work under Subtask 3.5. Additional high-energy D-D measurements for neutron energies in the 3-7 MeV range are planned using the Lawrence Livermore National Laboratory neutron generator.

Subtask 3.6 Develop De-Convolution Methodology for High-Energy Neutron Spectrometry The neutronresponse measurements from Subtask 3.5 are being used to benchmark neutron response spectral unfolding calculations in order to derive methods for inferring the incident neutron spectrum from the SiC response spectrum.

In summary, all scheduled milestones for years one were completed on schedule and all scheduled milestones for year two will be completed before the end of year two. All scheduled tasks for year two are on schedule.

\subsection{Plans for Year Three}

During the third year of this program, the neutron spectrum unfolding (de-convolution) methods will be completed. Additional neutron irradiations will be carried out using the high-energy D-D neutron source at the DOE Lawrence Livermore National Laboratory. This source produces mono-energetic neutrons in the 3-7 MeV range. Many of the peaks observed in $14-\mathrm{MeV}$ neutron irradiations will also be observed at the higher end of the 3-7 MeV range, providing further information to characterize the neutron response at higher energies. The Si detector measurements to determine the range straggling contribution to the measured SiC energy resolution will be completed. Long-term gamma-ray irradiation and testing of SiC detectors will be continued to a total cumulative gamma-ray dose of 4E09 Rad. Mixed-field response testing will be carried out for alpha particles, X-rays and low-energy gamma rays, and fast neutrons using the Westinghouse Gamma Hot Cell. Measurements with SiC detectors will be carried out at a DOE cleanup site. Initial discussions are underway with both the Savannah River Laboratory and the Idaho National Engineering and Environmental Laboratory. In the former case, waste tank actinide monitoring applications are being discussed. In the latter case, applications related to decommissioning of waste cleanup sites are being discussed. 


\subsection{Highlights of Progress During Year-Two}

1. Testing of a new class of large-volume SiC detectors has continued. 2. Excellent alpha-particle energy linearity and peak resolution for these $\mathrm{SiC}$ detectors has been demonstrated. Recent results demonstrate that energy resolution comparable to the best obtainable with conventional silicon detectors can be obtained with $\mathrm{SiC}$ detectors. 3. Only negligible $\mathrm{SiC}$ detector response changes have been observed after a cumulative gamma-ray exposure of 1.4E09 Rad. Silicon detectors show significant performance degradation at E06 Rad. 4. Fast-neutron response spectra show detailed energy information from neutroninduced reactions in both silicon and carbon. These results indicate that $\mathrm{SiC}$ detectors should prove useful for neutron energy spectrometry applications in elevated-temperature, high-radiation environments.

These results and the demonstrated capability of SiC detectors to operate for prolonged times in elevatedtemperature and high-radiation measurement environments will lead to many potential applications in DOE environmental characterization and decommissioning applications.

DELIVERABLES: The following papers were presented:

1. "The Fast Neutron Response of Silicon Carbide Semiconductor Radiation Detectors" , F. H. Ruddy, A. R. Dulloo, J. G. Seidel, M. K. Das, S-H Ryu, and A Agarwal, 2004 IEEE Nuclear Science Symposium and Medical Imaging Conference, October, 2004 (to be published in IEEE Transactions on Nuclear Science).

2. "Fast Neutron Dosimetry and Spectrometry Using Silicon Carbide Semiconductor Radiation Detectors", F. H. Ruddy, J. G. Seidel, and A. R. Dulloo, 12th International Symposium on Reactor Dosimetry, Gatlinburg, 2004 (to be published in the Journal of ASTM International).

3. "The Effects of Intense Gamma-Irradiation on the Alpha-Particle Response of Silicon Carbide Semiconductor Radiation Detectors”,F. H. Ruddy and J. G. Seidel, Sixth International Topical Meeting on Industrial Radiation and Radioisotope Measurement Applications (IRRMA-6), June, 2005 (to be published in Nuclear Instruments \& Methods B).

The following paper will be presented at the IEEE 2004 Nuclear Science Symposium in October 2004: "High-Resolution Alpha-Particle Spectrometry Using Silicon Carbide Semiconductor Detectors", F. H. Ruddy, J. G. Seidel, H. Chen, A. R. Dulloo, and S.-H. Ryu (Proceedings to be published in IEEE Transactions on Nuclear Science).

A Nuclear Engineering Department seminar highlighting results of this program was presented at the University of Florida in January, 2005.

A Nuclear Engineering Department seminar highlighting results of this program is scheduled to be presented at North Carolina State University in November, 2005. 